\title{
Effect of surface chemistry on Islet Amyloid Polypeptide conformation
}

\author{
David L. Cheung* \\ School of Chemistry, National University of Ireland Galway, Galway, Ireland
}

\begin{abstract}
The formation of dense, linear arrays (fibrils) by biomolecules is the hallmark of a number of degenerative diseases, such as Alzheimer's and type-2 diabetes. Protein fibrils have also attracted interest as building blocks for new materials. It has long been recognised that surfaces can affect the fibrillation process. Recent work on the model fibril forming protein human islet amyloid polypeptide (hIAPP) has shown that while the protein concentration is highest at hydrophobic surfaces, the rate of fibril formation is lower than on other surfaces. To understand this, replica exchange molecular dynamics simulations were used to investigate the conformations that hIAPP adopts on surfaces of different hydrophobicity. The hydrophobic surface stabilizes $\alpha$-helical structures, which are significantly different to those found on the hydrophilic surface and in bulk solution. There is also a greatly reduced conformational ensemble on the hydrophobic surface, due to long-lived contacts between hydrophobic residues on the protein and the surface. This new microscopic information will help us determine the mechanism of the enhancement of fibril formation on surfaces and provides new insight into the effect of nanointerfaces and protein conformation.
\end{abstract}




\section{INTRODUCTION}

Understanding the formation of amyloid fibrils, dense, linear protein arrays, is a major challenge in biophysics ${ }^{1}$. They are formed by a wide range of proteins and first gained attention due to their involvement in Alziehmer's disease and subsequently amyloids have been implicated in over 30 different diseases, including Alzheimer's disease, Parkinson's disease, and type-II diabetes. Amyloids have been shown to have functional roles in biology, which include the formation of biofilms ${ }^{2}$, mediating adsorption onto surfaces ${ }^{3}$, and storage of hormones ${ }^{4}$. These functional amyloids often take advantage of their excellent mechanical and material properties. Amyloids are also being harnessed in materials science, in applications such as adhesives or biosensors ${ }^{5}$.

While fibrils can be formed by globular proteins, many of the most important examples of amyloid forming proteins are intrinsically disordered proteins (IDPs) such as amyloid beta, islet amyloid polypeptide (IAPP), and alpha-synuclein. In monomeric form IDPs are typically extended and flexible, so their aggregation into $\beta$-strand rich fibrils requires significant changes in conformation. The mechanism by which protein fibrils form is still unclear, with questions regarding the conformational changes the proteins adopt on going to the fibril structure and the role of oligomeric species still unanswered. Understanding the fibrillation process is important in developing treatments for amyloid diseases, in particular as it is currently thought that in some cases the toxic species are small oligomers rather than the fibrils themselves ${ }^{6}$. As such, knowledge of how filbrillation depends on protein environment is of significant interest.

The formation of protein fibrils is known to be affected by surfaces and interfaces ${ }^{7,8}$. As they are intrinsically amphiphilic, proteins often adsorb onto surfaces so the local concentration can be higher there than in bulk solution, which typically leads to an increase in the aggregation rate. Surfaces can also change protein conformation, due to both specific and non-specific protein-surface interactions. For IDPs this can be particularly pronounced as the surface can change the ensemble of structures adopted by the protein. The effect of this on fibril formation, however, is not universal and depends on the properties of both the protein and the surface ${ }^{7}$. In particular, the fibrillation of IAPP has also shown to be dependent on the chemistry of the surface, with formation of fibrils being retarded on hydrophobic surfaces compared to hydrophilic surfaces, despite the higher protein concentration on the 
hydrophobic surfaces ${ }^{9-11}$. This decoupling of protein concentration and fibrillation suggests that changes to the protein conformation on surfaces play a key role in determining the fibrillation process. Fibril formation (and the formation of higher order structures) is also dependent on the mobility of proteins on the surface ${ }^{8}$.

Understanding the conformation of proteins on surfaces is experimentally challenging due to the small length and time scales involved. While there are a number of techniques that can investigate the amount of protein on a surface and the layer thickness (e.g. quartz crystal microbalance (QCM), ellipsometry, X-ray and neutron reflectivity), information on protein structure can be harder to determine. High-speed AFM $^{12}$ can be used to investigate the structures of larger proteins and structures formed by aggregation of smaller ones (e.g. amyloid beta $\left.{ }^{13}\right)$. Recently sum frequency generation ${ }^{14}$ (SFG) has emerged as a powerful tool for the investigation of protein structure on surfaces. This has been used to to investigate the surface structure of a number of proteins, including fibril forming proteins such as IAPP ${ }^{15,16}$ and amyloid beta ${ }^{17}$.

Due to its ability to directly access microscopic length scales molecular dynamics (MD) simulations is well suited to studying protein conformation on surfaces ${ }^{18}$. MD simulations have been extensively used to investigate protein adsorption onto surfaces, giving information on factors such as the role of bound water ${ }^{19}$, identifying residues responsible for driving adsorption $^{20,21}$, and determining adsorption energies ${ }^{22}$. In particular MD simulation has been applied to investigate a number of amyloidogenic proteins, such as amyloid beta ${ }^{23}$, IAPP $^{24}$, and model peptides ${ }^{25-27}$, on surfaces. These have shown that the conformations adopted by IDPs depend on both surface chemistry and surface structure.

As they exist in a range of different structures rather than a single well-defined native state, accurately modelling IDPs is more challenging than for globular proteins. To comprehensively sample the conformational ensemble of IDPs the use of enhanced sampling methods, such as metadynamics ${ }^{28}$ or replica exchange molecular dynamics ${ }^{29}$ (REMD), are often used. These have been shown to be effective for the investigation of IDPs, both in bulk solution $^{30}$ and increasingly at surfaces and interfaces ${ }^{31}$. Metadynamics and replica exchange simulations have been applied to the study of amyloid beta onto gold surfaces ${ }^{17,32}$, showing the the surface changes the conformational ensemble and demonstrating the differing effect of surface adsorption on the full protein and the central amyloidogenic fragment. Enhanced sampling methods have also been used for studying biominerialization peptides on inorganic 
surfaces, investigating the facet selectivity of these ${ }^{33,34}$.

As the chemistry of surfaces has been shown to affect the fibrillation process it is useful to understand the conformations of amyloidogenic proteins on surfaces of different chemistry. In this paper the structure of hIAPP (human islet amyloid polypeptide, Figure 1), a typical amyloidogenic polypeptide, on surfaces of different hydrohobicity is investigated. To enhance the sampling of its conformations REMD, specifically replica exchange with solute tempering (REST), simulations are used. On hydrophobic surfaces hIAPP is found to behave differently to hydrophilic surfaces and in bulk solution, exhibiting structures that are significantly different and having a significantly reduced conformational ensemble. This illustrates that changes to surface chemistry can have a large effect on the conformation of single molecules, which will change their subsequent aggregation and assembly on surfaces.
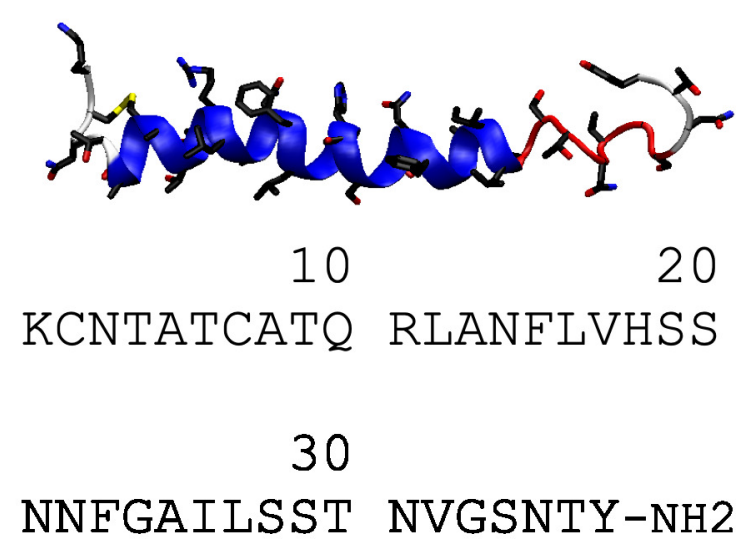

FIG. 1. Structure (top) and sequence (bottom) of human islet amyloid polypeptide (hIAPP).

\section{MODEL AND METHDOLOGY}

\section{A. Simulated system}

For all simulations the system contained a single human IAPP protein, with the initial structure taken from the NMR structure ${ }^{35}$ (pdb:2kb8). Following the biological structure the C-terminus was amidated and the charges on the polarizable residues (Arg, Asp, Glu, His, and Lys) were set appropriate for $\mathrm{pH}=7$. The surfaces consisted of an an alkylthiol selfassembled monolayer, specifically containing either hydrophobic $\left(\mathrm{R}=\mathrm{CH}_{3}\right)$ or hydrophilic 
$(\mathrm{R}=\mathrm{OH})$ ligands, denoted as SAMch3 and SAMoh. The chains were arranged in the $\sqrt{3} \times$ $\sqrt{3}$ R3 geometry, using structures provided by the Latour research group (https://cecas . clemson.edu/latourlabs/Jmol/Surfaces.html), with $320(20 \times 16)$ chains in total. To mimic the effect of strong anchoring onto an underlying surface the terminal sulfur and hydrogen atoms of the chains was fixed.

All systems were constructed using standard Gromacs utilities. For the surface simulations the protein was initially placed $20 \AA$ from the surface, where the surface was defined as the average $z$ position of the terminal heavy atoms. All systems were solvated and counterions were added to neutralise the protein. The systems were initially energy minimised using the steepest descent algorithm followed by short (20 ps) NVT simulations (at $300 \mathrm{~K}$ ), first with the positions of the heavy atoms in the protein restrained to their initial positions by harmonic potentials (with force constant $2.4 \mathrm{kcal} \mathrm{mol}^{-1} \AA^{-2}$ ), then without the position restraints. A short (20 ps) NpT-simulation was then performed for the bulk solution.

To describe the inter- and intramolecular interactions the Charmm $36 \mathrm{~m}^{36,37}$ force field along with the charmm-TIP3P ${ }^{38,39}$ water model was used. Parameters for the alkylthiol chains were taken from the charmm general force field ${ }^{40}$.

\section{B. Simulation method}

In order to enhance sampling of protein conformations replica exchange with solute tempering (REST) was employed ${ }^{41,42}$. This is a variation on replica exchange molecular dynamics ${ }^{43}$, where the temperature varies only for a subset of the system, in this case the protein. The temperature scaling was performed by scaling the protein-protein and proteinsolvent interactions by a factor depending on the effective temperature. Specifically the potential energy was given by ${ }^{42}$

$$
E_{i}=\beta_{i} E_{p p}+\beta_{i}^{1 / 2} E_{p s}+E_{s s}
$$

where $E_{p p}, E_{p s}$, and $E_{s s}$ are the protein-protein, protein-solvent, and solvent-solvent interaction and the scaling factor $\beta_{i}=T_{0} / T_{i}$. For all systems the effective temperature was in the range $300 \mathrm{~K}$ to $440 \mathrm{~K}$, with 12 replicas used for the full protein. The scaling factors and effective temperatures for the different replicas were 1 (300 K), 0.966 (310.6 K), 0.933 (321.6 K), 0.901 (333 K), $0.870(344.8 \mathrm{~K}), 0.840$ (357 K), 0.811 (369.7 K), $0.784(382.8 \mathrm{~K}), 0.757$ 
(396.4 K), $0.731(410.4 \mathrm{~K}), 0.706$ (424.9 K), and 0.682 (440 K). Exchange attempts between neighbouring replicas were attempted 500 time steps (1 ps). Transitions between different temperatures and acceptance rates are given in the supporting information (Figure S1 and Table S1).

Surface simulations were performed in the $N V T$-ensemble with the temperature controlled using a velocity rescaling algorithm ${ }^{44}$, with a relaxation time of 0.2 ps. Bulk simulations were performed in the $N p T$-ensemble using the Parrinello-Rahman barostat ${ }^{45}$ (relaxation time $2 \mathrm{ps}$ ) to control the pressure. All simulations were performed at a temperature of $300 \mathrm{~K}$ and the bulk simulations were performed at a pressure of $1 \mathrm{~atm}$. For the surface simulations the system was periodic in the $x$ and $y$ directions. To contain the system in the $z$-direction walls, interacting through the integrated 9-3 LJ potential were used. The bulk simulations were periodic in all directions. A cutoff of $12 \AA$ was used for the van der Waals and short-range electrostatic interactions. Long-range electrostatic interactions were evaluated using a Particle Mesh Ewald ${ }^{46}$ sum with a Fourier spacing of $0.16 \mathrm{~nm}$. Reciprocal space grids of $36 \times 36 \times 36$ (bulk solution) and $40 \times 40 \times 160$ (surface) were used. The equations of motion were integrated using a timestep of $2 \mathrm{fs}$, with the LINCS algorithm use to constrain bond lengths ${ }^{47}$. Simulations were performed using the Gromacs simulation package (version 4.6.7 ${ }^{48-50}$, using the PLUMED library ${ }^{51}$ to implement REST simulations.

Simulation lengths were $400 \mathrm{~ns}$ for the surface simulations and $620 \mathrm{~ns}$ for the bulk simulation. Equilibration was judged from considering the number unique conformations found from cluster analysis (Figure S2), with equilibration being achieved once the number of low energy clusters (with free energy within $3 \mathrm{kcal} \mathrm{mol}^{-1}$ of the most populated cluster) had plateaued. Following this the simulations were run for a further 100 ns, with analysis performed over this period (unless otherwise stated).

\section{Analysis}

Analysis of the simulations was performed using a combination of standard gromacs utilities, custom-written python scripts using the MDAnalysis library ${ }^{52}$, and VMD (visual molecular dynamics ${ }^{53}$. The secondary structure analysis was performed using the STRIDE algorithm ${ }^{54}$. Similarity to helices was also analysed through determining the number of 
$\alpha$-helical and 3/10-helical hydrogen bonds ${ }^{22}$

$$
\begin{aligned}
N_{\alpha-H B} & =\sum_{i=1}^{N_{H B}} \frac{1-\left(r_{i} / r_{0}\right)^{n}}{1-\left(r_{i} / r_{0}\right)^{m}} \\
N_{3 / 10-H B} & =\sum_{i=1}^{N_{H B}} \frac{1-\left(r_{i} / r_{0}\right)^{n}}{1-\left(r_{i} / r_{0}\right)^{m}}
\end{aligned}
$$

where $r_{0}=2.5 \AA, n=8, m=12$, and the sums run over all potential $\alpha$-helical (equation 2a) and 3/10-helical (equation 2b) hydrogen bonds, i.e. between backbone carbonyl oxygens and amine hydrogens separated by 4 or 3 residues respectively. The similarity to $\beta$-strands was analysed through the dihedral offset function

$$
D H=\frac{1}{2} \sum_{i=1}^{N-1}\left(1+\cos \left(\phi_{i}-\phi_{\text {ref }}\right)\right)+\left(1+\cos \left(\psi_{i}-\psi_{\text {ref }}\right)\right) .
$$

where the sum runs over the $\phi$ and $\psi$ angles of the protein residues and the reference angles are $\phi_{\text {ref }}=-2.36 \mathrm{rad}$ and $\psi_{\text {ref }}=2.36 \mathrm{rad}$, corresponding to an ideal $\beta$-strand with alternating residues on opposite sides of the protein backbone.

Protein size was characterised through the radius of gyration

$$
R_{g}^{2}=\frac{1}{N} \sum_{i=1}^{N}\left(\boldsymbol{r}_{i}-\boldsymbol{r}^{\mathrm{com}}\right)^{2}
$$

where $\boldsymbol{r}_{i}$ is the position of the $i$ th atom and $\boldsymbol{r}^{\text {com }}$ is the protein centre of mass and the sum runs over atoms in the protein and the eigenvalues of the gyration tensor

$$
G_{\alpha \beta}^{2}=\frac{1}{N} \sum_{i=1}^{N}\left(r_{i \alpha}-r_{\alpha}^{c o m}\right)\left(r_{\beta}-r_{\beta}^{c o m}\right), \alpha, \beta=x, y, z .
$$

Formation of compact structures can also be investigated through the number of contacts between $\mathrm{C} \gamma$ atoms calculated using

$$
N_{C \gamma}=\sum_{i}^{N} \sum_{j>i}^{N} f_{\text {switch }}\left(r_{i j}\right)
$$

where the double sum runs over $\mathrm{C} \gamma$ atoms on different residues and the switching function is given by

$$
f_{\text {switch }}(r)=\frac{1-\left(r / r_{0}\right)^{n}}{1-\left(r / r_{0}\right)^{m}}
$$

where $m=12, n=8$ and $r_{0}=4.5 \AA$. Contacts between $\mathrm{C} \alpha$ atoms $\left(N_{C \gamma}\right)$ are calculated similarly (with $r_{0}=8 \AA$ ). The conformational ensemble was analysed from a cluster analysis, using the method of Daura et a $\hbar^{55}$, using a cut off of $3 \AA$. Unless otherwise stated analysis was performed for the $\beta=1$ replica (the only physically relevant one). 


\section{RESULTS}

\section{A. Surfaces perturb protein structure}

Due to the differences in the fibrillation behaviour it may be expected that the conformation of IAPP on different surfaces may be quite different. Shown in Figure 2(a) are the secondary structures on both different surfaces and in bulk solution. On the hydrophobic surface the protein largely adopts an $\alpha$-helical structure, containing residues N3-S28 which remains stable for the majority of the simulation. Formation of helical conformations at hydrophobic surfaces and interfaces has been seen in previous simulation ${ }^{31,56}$ studies of intrinsically disordered proteins. The only region of the protein that varies from this is a small section near the C-terminus that is largely $\beta$-strand and turn. On the SAMoh surface and in bulk solution the secondary structure is more varied. Typically the $\alpha$-helical content is somewhat lower and largely concentrated in the first half of the protein (corresponding to the membrane binding domain), while the remainder of the protein largely consists of turn or random coil. There are also conformations which have high $\beta$-strand content, which may correspond to precursors to fibrils. The higher preponderance of these on the SAMoh surface compared to the SAMch3 surface, suggests that the hydrophilic surface may more easily template the formation of fibrils.

More information on the secondary structure can be seen from the secondary structure propensity (Figure 2(b)). On the SAMch3 surface the first three residues are completely disordered and the C-terminal region (T30-Y37) exhibit $\beta$-strand, turn, and coil structures.

Otherwise the protein is largely $\alpha$-helical, although residues S20-S29 has some propensity for $\beta$-strand formation. This structure is quite different to that found on the SAMoh and in bulk solution. In these cases the propensity for $\alpha$-helix formation is lower and the $\alpha$-helical region is smaller, consisting of residues T4-S20. This $\alpha$-helical domain is similar to that found in previous simulations studies of hIAPP ${ }^{57}$. The remainder of the protein is more variable, consisting of a mixture of $\beta$-strand, turn, and random coil. This variable region also shows differences between the SAMoh surface and bulk solution; on the hydrophilic surface there is a small additional region of helix (either $\alpha$-helix or 3/10-helix) which is absent in bulk solution.

The differences in the secondary structures of the protein on different surfaces can also 

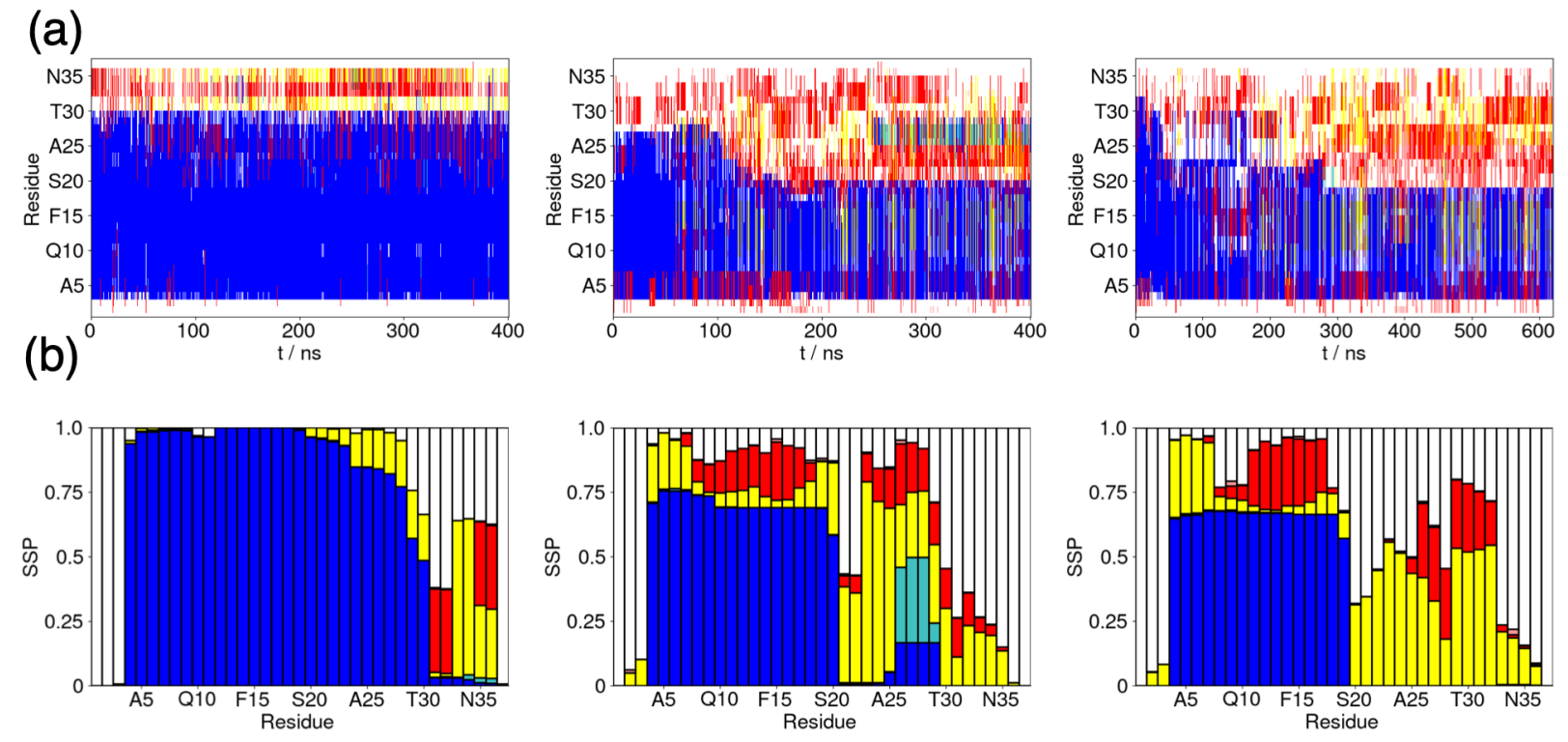

FIG. 2. (a) Variation of secondary structure with time for hIAPP for (left to right) SAMch3 surface, SAMoh surface, and bulk solution. $\alpha$-helix, $\beta$-strand, turn, 3/10-helix, and coil denoted by blue, yellow, red, cyan, and white respectively. (b) Secondary structure propensity for each residue (averaged over last $100 \mathrm{~ns}$ of simulations). Colours as in (a)

been seen through the number of hydrogen bonds and dihedral offset (Table I). Consistent with the secondary structure $N_{\alpha-H B}$ and $N_{3 / 10-H B}$ are significantly higher on the SAMch3 surface than on the SAMoh surface and in bulk solution, while $D H$ is lower on the SAMch3. While these are similar for the SAMoh surfaces and bulk solution, the number of hydrogen bonds is slightly higher and $D H$ slightly lower for the SAMoh surface compared to bulk solution, illustrating the change in structure between bulk solution and hydrophilic surface.

Surface adsorption also affects the protein tertiary structure. The radius of gyration increases on the SAMch3 surface. From the eigenvalues of the gyration tensor this is largely due to extensions along the long axis, due to the rigidity of the $\alpha$-helix. The other eigenvalues are slightly higher on the SAMoh surface and in bulk solution. The more compact structures can also be seen be considering the number of protein-protein contacts. Both $N_{C \alpha}$ and $N_{C \gamma}$ are lowest on the SAMch3 surface, consistent with the less compact structure compared to the other systems. These are larger on the SAMoh surface than in bulk solution, indicating that although the protein generally has similar structures in these environments some differences are found. From the $\mathrm{C}_{\alpha}$-contact maps (Figure 3) on the SAMch3 


\begin{tabular}{lccc}
\hline & SAMch3 & SAMoh & Solution \\
\hline$N_{\alpha-H B}$ & $19 \pm 3$ & $10 \pm 6$ & $8 \pm 5$ \\
$N_{3 / 10-H B}$ & $12 \pm 1$ & $8 \pm 4$ & $6 \pm 3$ \\
$D H$ & $31 \pm 2$ & $40 \pm 10$ & $44 \pm 9$ \\
\hline$R_{g} / \AA$ & $14.6 \pm 2.0$ & $11.1 \pm 2.0$ & $11.2 \pm 1.9$ \\
$G_{\text {max }} / \AA$ & $13.7 \pm 2.3$ & $9.3 \pm 2.3$ & $9.4 \pm 2.2$ \\
$G_{\text {mid }} / \AA$ & $4.2 \pm 0.8$ & $4.8 \pm 0.7$ & $4.9 \pm 0.6$ \\
$G_{\text {min }} / \AA$ & $2.8 \pm 0.2$ & $3.6 \pm 0.4$ & $3.7 \pm 0.4$ \\
\hline$N_{C \alpha}$ & $190 \pm 14$ & $216 \pm 23$ & $208 \pm 21$ \\
$N_{C \gamma}$ & $23 \pm 3$ & $32 \pm 6$ & $28 \pm 4$ \\
\hline \multicolumn{4}{c}{}
\end{tabular}

TABLE I. Measures of protein structure. Uncertainties estimated from standard deviation
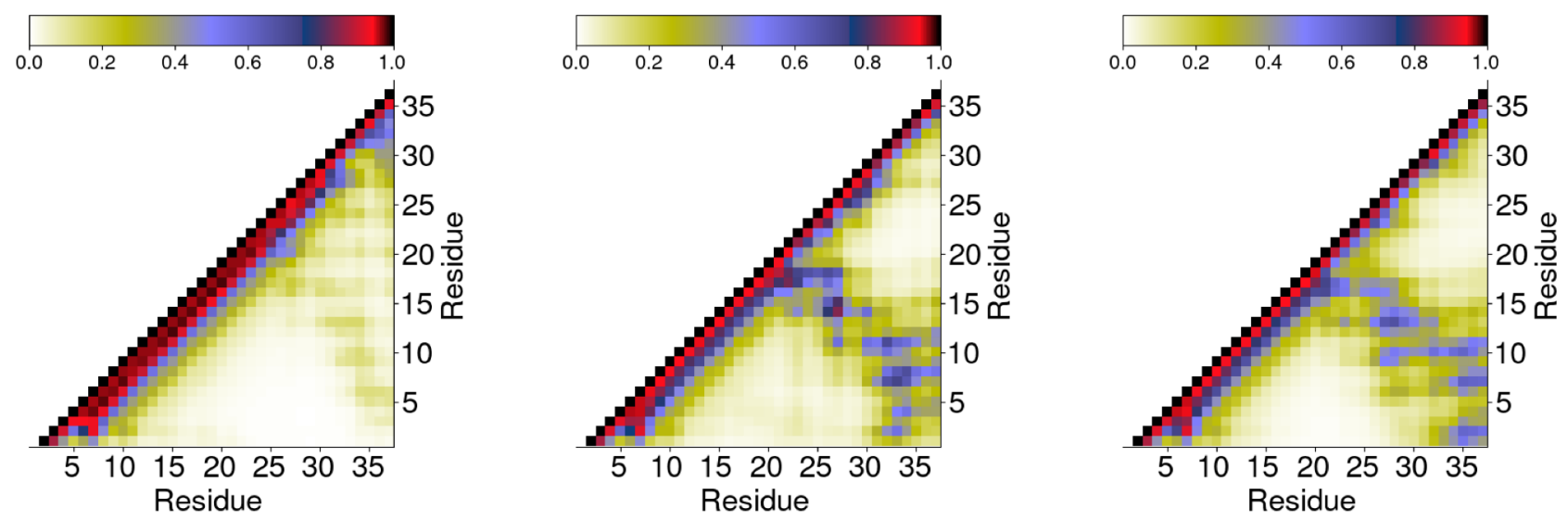

FIG. 3. $\mathrm{C}_{\alpha}$ contact maps for hIAPP. From left to right SAMch3, SAMoh, and solution.

surface contacts are largely between closely separated residues with only a low probability of contacts between more distant (in sequence space) residues. On the SAMoh surface and in bulk solution contacts between more distant residues, in particular contacts between the $\mathrm{N}$ - and C-termini are found. 


\section{B. Adsorption changes the conformational ensemble in a surface dependent man-} ner

Due to its intrinsic disorder hIAPP exhibits an ensemble of different conformations, both on surfaces and in bulk solution. Using cluster analysis ${ }^{55}$ (on the last 100 ns of each simulation) the unique conformations are determined. Even from consideration of the number of unique conformations found clear differences between the hydrophobic surface and other systems are found; for the SAMch3 surface 17 unique conformations were seen, whereas 260 and 315 were found for the SAMoh surface and bulk solution respectively. This suggests that adsorption onto the hydrophobic surface greatly restricts the possible conformations of the protein. The wide range of conformations adopted on the hydrophilic surface is also consistent with formation of different fibril polymorphs seen on hydrogen bonding surfaces ${ }^{58}$.

Shown in Figure 4 are the most common conformations (consisting of $75 \%$ of all conformations found) for hIAPP on surfaces and in bulk solution. From the cluster analysis the structures adopted on the SAMch3 surface are significantly different to those typically found on the SAMoh surface and bulk solution. In particular the most common conformations in the SAMch3 surface have a high $\alpha$-helical content (Figure 2). The conformations on the SAMoh surface and in bulk solution are more similar to each other. For both cases the most common conformations have high $\alpha$-helix content but structures with high $\beta$-strand content, similar to structures in fibrils, are seen.

The free energy of the different clusters (relative to the most populous cluster) can be estimated as

$$
\Delta F=-R T \ln \frac{P_{i}}{P_{1}}
$$

where $P_{i}$ is the probability of the $i$ th cluster. For the SAMch3 surface the free energies of the first two clusters are similar to each other (Figure 5), with another three clusters within $\sim 2 k_{\mathrm{B}} T$, reflecting the similarity between the clusters found for the SAMch3 surface. There is a larger difference in free energy for the lowest energy conformations for the SAMoh surface and bulk solution, indicative of the larger difference in structures.

To qualify how surface adsorption affects the conformational ensemble the population of solution-like conformations was found. For each (saved) conformation from the surface simulation the $\mathrm{C}_{\alpha}-\mathrm{C}_{\alpha}$ distance root-mean squared difference (DRMSD) with each solution

cluster was determined, with the conformations matching if DRMSD $\leq 3 \AA$. On the SAMch3 

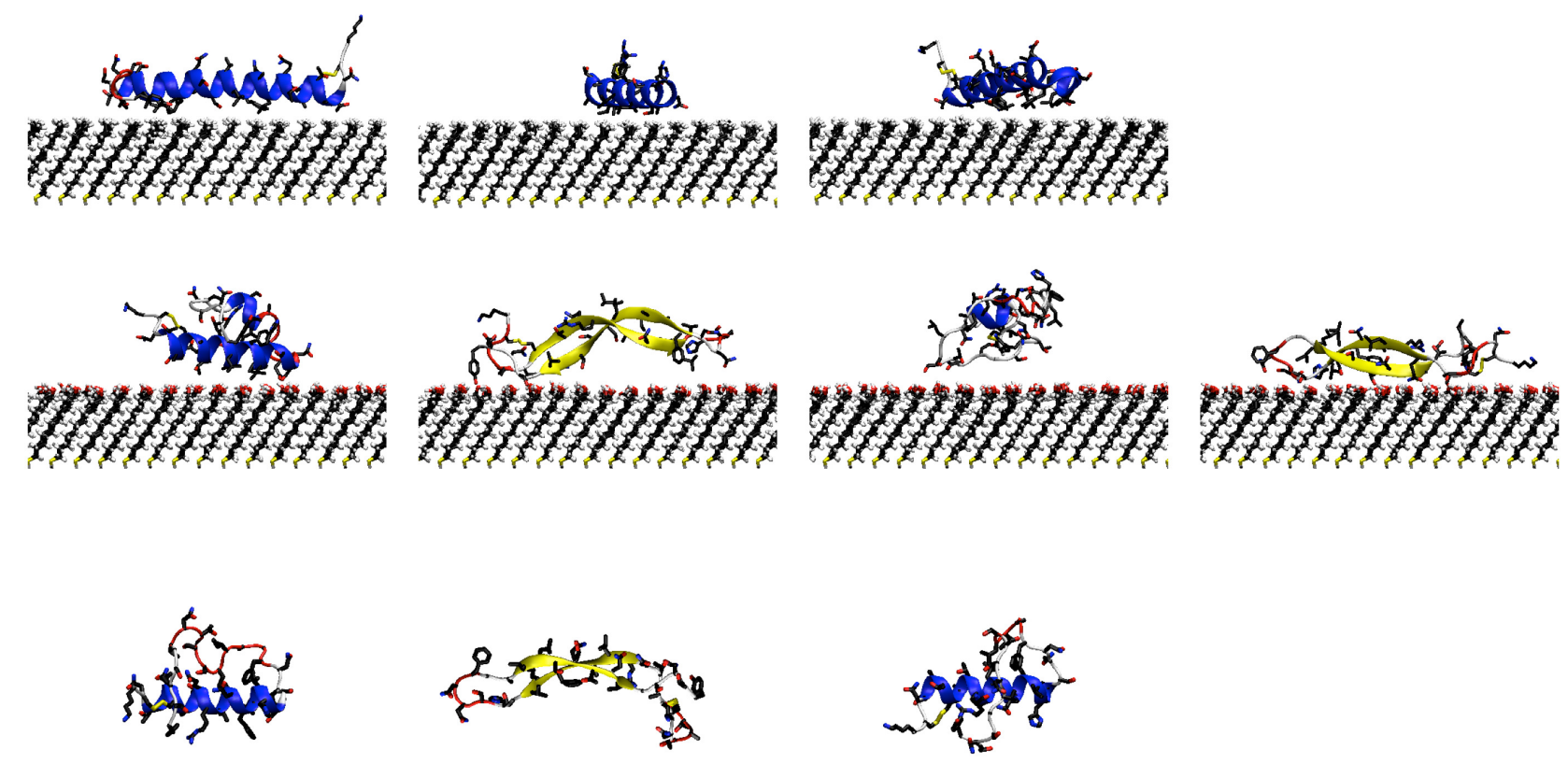

FIG. 4. Snapshots of most common conformations identified using cluster analysis for hIAPP. Top shows structures on SAMch3 surface, middle SAMoh surface, and bottom bulk solution.

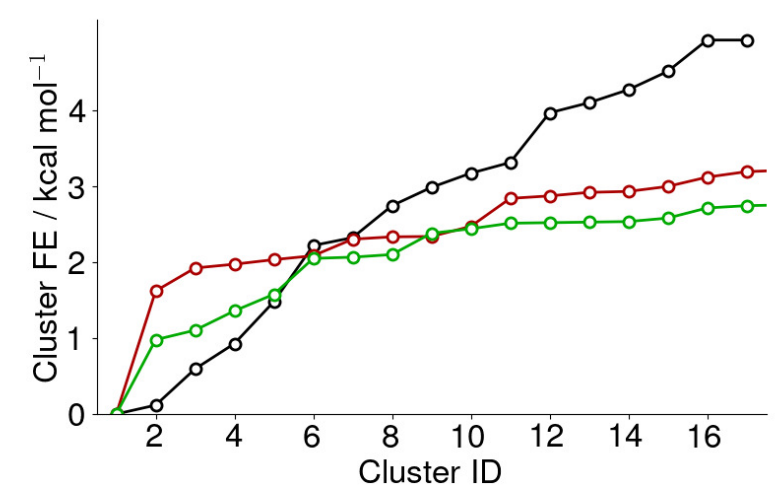

FIG. 5. Free energies of clusters. Black, red, and green denote SAMch3, SAMoh, and bulk solution.

surface only two solution conformations are found in the simulations (Figure 6 and Table S2); these are both found with low $(<10 \%)$ probability and correspond to relatively high free energy conformations. Otherwise for the majority $(>85 \%)$ of the simulation the structures found for the hydrophobic surface do not correspond to any of the conformations found in the bulk solution, suggesting that on the hydrophobic surface hIAPP adopts qualitatively 
different conformations than in bulk solution. Similar induced fit behaviour, where the surface induces the formation of different structures, has been seen for amyloid beta on gold surfaces $^{32}$.

On the SAMoh surface more solution-like conformations are found, corresponding to $\sim 87 \%$ of the simulation. However, these generally these do not correspond to the most likely conformations in bulk solution; in solution the conformations found on the hydrophilic

surface typically have free energies $>3.8 \mathrm{kcal} \mathrm{mol}^{-1}$ compared to the most likely conformation. This shows that on the SAMoh surface hIAPP exhibits conformational selection-like behaviour, where the adsorbed polypeptide adopts similar conformations to bulk solution, albeit with different probabilities. Similar behaviour has been shown for the amyloid beta(1622) fragment on gold surfaces ${ }^{17}$. The most common conformation, which corresponds to about $61 \%$ of the simulation, is largely $\alpha$-helical, but other $\beta$-strand rich conformations are found.

\section{Surface hydrophobicity favours the formation of helical over fibril-like confor- mations}

In order to investigate the effect of different environments on the formation of fibrils and other supramolecular aggregates it is instructive to compare the similarity between the simulation structures and experimental structures of IAPP in different environments. Shown in Figure 7 are the histograms of the $\mathrm{C}_{\alpha}-\mathrm{C}_{\alpha}$ DRMSD between the simulation conformations and experimental helical ${ }^{35}$ and fibril structures ${ }^{59}$ for hIAPP. For the SAMch3 surface there is a peak in $P(D R M S D)$ for the helical structure at $D R M S D \sim 2-3 \AA$, indicating a high probability of finding states similar to the experimental helical structure. This suggests that on the hydrophbic surface hIAPP behaves in a similar manner to the micelle environment. In contrast for the fibrillar structure $D R M S D>10 \AA$ showing there is little similarity with the fibril structure.

There is significantly less similarity to either the fibril or helical structure for the SAMoh surface and in bulk solution. Relative to the fibrillar structure these both have a small peak in $P(D R M S D)$ at $D R M S D \sim 6 \AA$, suggesting some fibril-like structures forming in these environments. Note that the experimental fibril structure is taken from a fibril so may not be representative of the structures formed by single molecules prior to fibril formation. 


\section{SAMch3}

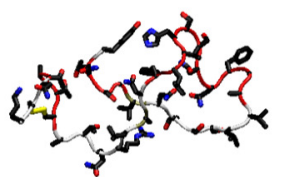

Cluster ID 263 $\mathrm{P}=0.095, \mathrm{FE}=5.16$

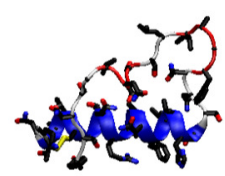

Cluster ID 73

$\mathrm{P}=0.050, \mathrm{FE}=3.85$

\section{SAMoh}

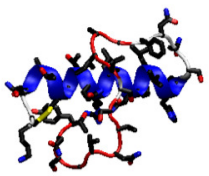

Cluster ID 215 $\mathrm{P}=0.613, \mathrm{FE}=5.16$

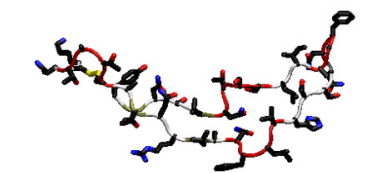

Cluster ID 305 $\mathrm{P}=0.033, \mathrm{FE}=5.16$

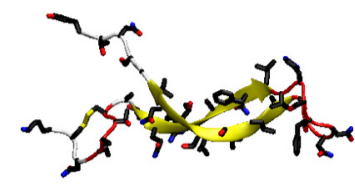

Cluster ID 77 $P=0.040, F E=3.85$

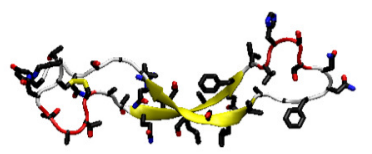

Cluster ID 223

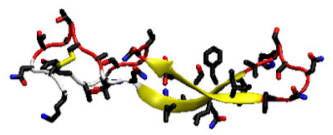

Cluster ID 309

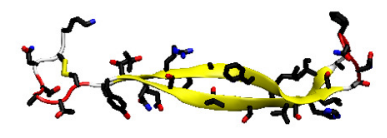

Cluster ID 297

FIG. 6. Solution-like conformations found in surface simulations. $P$ is the probability of that conformation being found in the surface simulations. $F E$ is the free energy of that conformation in the bulk simulations relative to the most most populated conformation (in $\mathrm{kcal} \mathrm{mol}^{-1}$.)

There is a larger difference to the experimental helical structure compared to the SAMch3 surface.

Both the experimental fibril structure and fibril-like structures found on the SAMoh surface and in bulk solution all contain two $\beta$-strand segments. In the experimental structure these contain residues A8-V17 and S28-T36. On the SAMoh surface the $\beta$-strand regions contain residues C7-L16 and F23-V32 (from conformations corresponding to cluster 2) or T9-V17 and I26-V34 (for clusters 4 and 6), while in bulk solution these are residues R11V17 and I26-V32. The overlap with the first $\beta$-strand, for both SAMoh and bulk solution, suggests that the formation of this strand may be the initial step in the formation of fibril-like conformations. 

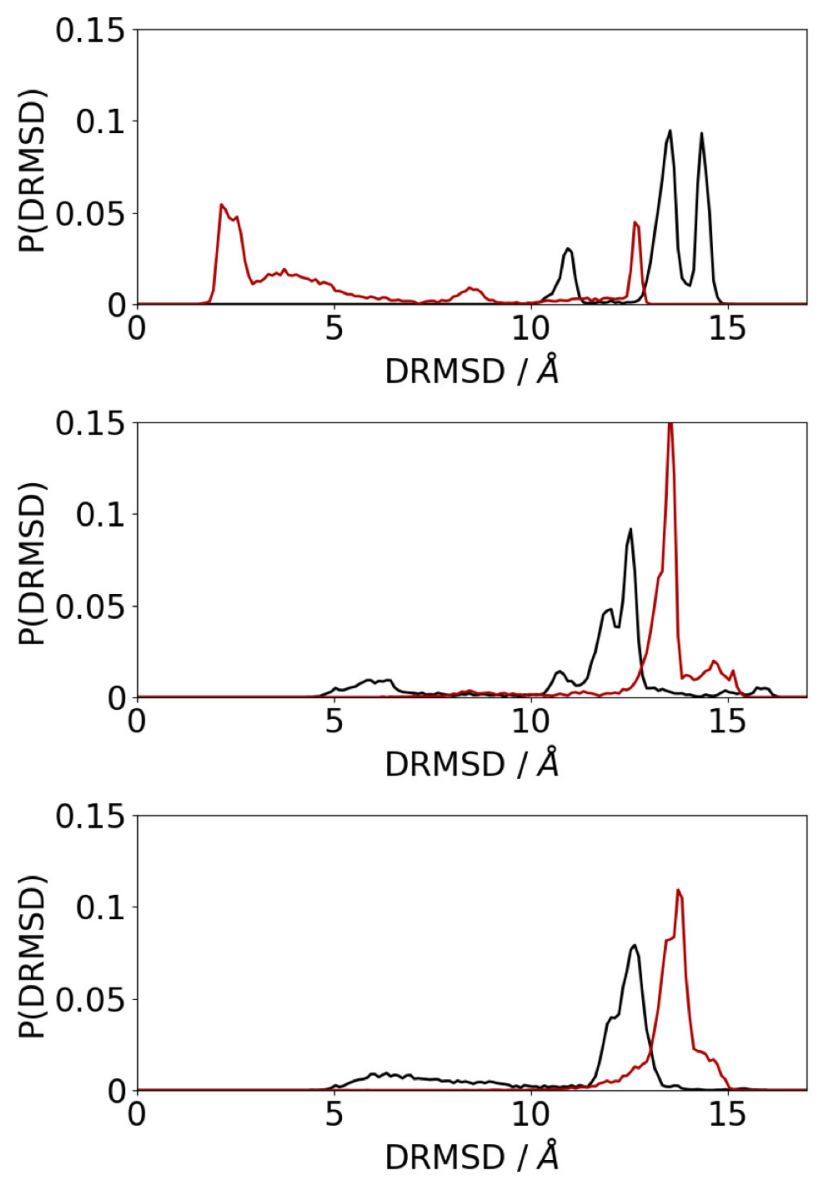

FIG. 7. Probability histograms for DRMSD between simulation and experimental structures for SAMch3 (top), SAMoh (middle), and solution (bottom). $P(D R M S D)$ to fibril and helix structures denoted by black and red respectively.

\section{How do different surfaces drive structural change?}

The different conformations adopted by hIAPP on hydrophobic and hydrophilic surfaces may be understood in terms of the different interactions between the polypeptide and the surfaces. For both surfaces residues near the centre of hIAPP are typically closer to the surface (Figure 8(a)). This differs from previous studies of hIAPP on citrate-coated gold surfaces $^{24}$, where the N-terminus was found to be closest to the surface. In that case adsorption was driven by electrostatic interactions between the negatively-charged citrate and positively charged lysine residue. Recent work on the hIAPP20-29 fragment with different terminal charges ${ }^{60}$ has demonstrated the complex role that electrostatic interactions can 
(a)

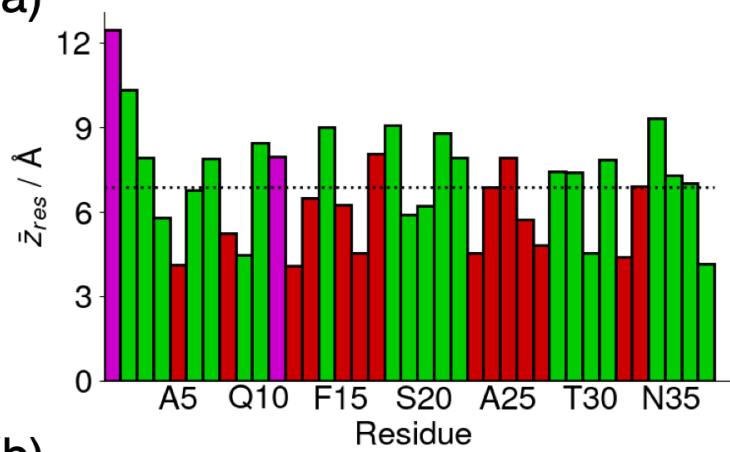

(b)

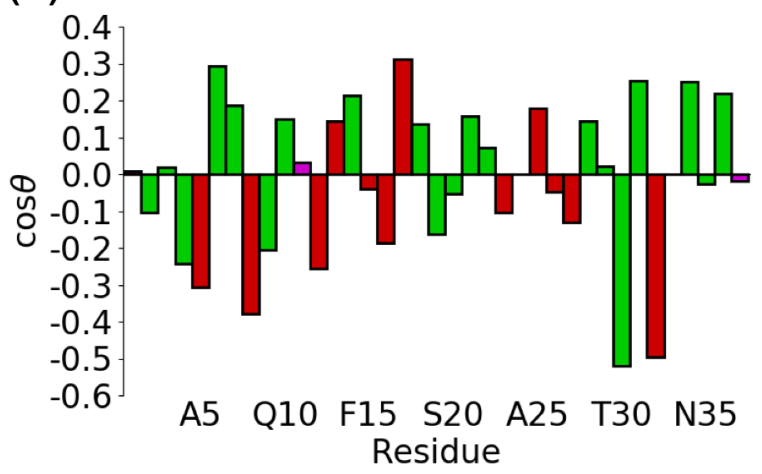

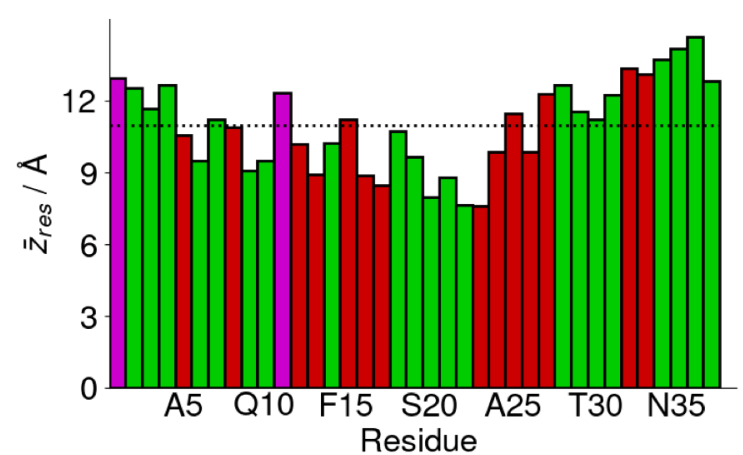

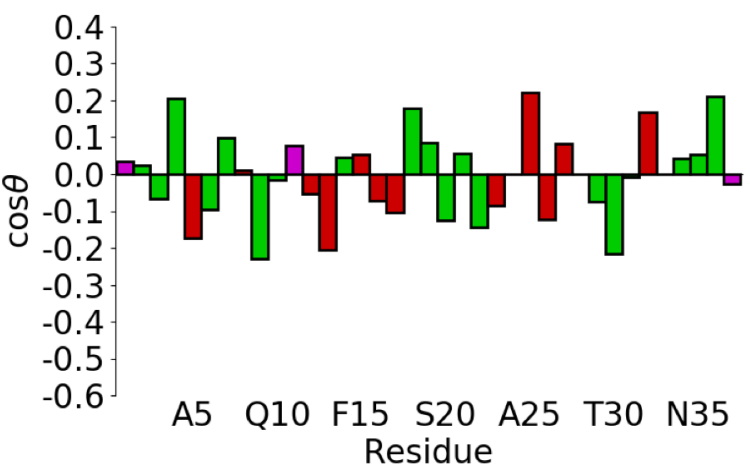

FIG. 8. (a) Average residue-surface separations for hIAPP on SAMch3 (left) and SAMoh (right) surfaces. Red, green, and magenta denote hydrophobic, polar, and positively-charged residues respectively. Dotted line denotes average hIAPP centre-of-mass-surface separation. (b) Sidechain orientations for hIAPP on (left) SAMch3 and (right) SAMoh surfaces. Colours as in (a)

play in determine polypeptide structure on surfaces. On the SAMch3 surface hydrophobic residues are, as is intuitively obvious, more likely to be in contact with the surface with the average residue-surface separations for the hydrophobic residues being smaller than for hydrophilic residues (Figure 8(a)). The pattern of the residue-surface separation is also reminiscent of that of an $\alpha$-helix, in agreement with the secondary structure analysis (Figure 2). In contrast on the hydrophilic SAMoh surface there is less difference between the hydrophobic and hydrophilic residues. Notably the region closest to the surface (S19-I26) largely contains hydrophilic residues suggesting surface adsorption may be mediated through hydrogen bonding with the surface. The typical residue-surface separations are also larger for the SAMoh surface compared to the SAMch3 surface.

Different interactions between the residues and surfaces also leads to changes in the orientations of the sidechains. Shown in Figure $8(\mathrm{~b})$ are the average of $\cos \theta$ (where $\theta$ is 
the angle between the vector joining the $\mathrm{C}_{\alpha}$ atom and the terminal heavy atom in each sidechain and the $z$-axis). For the SAMch3 surface these values are typically larger in magnitude, indicating a stronger ordering effect of the surface. The hydrophobic residues typically have $\cos \theta<0$ showing that these typically point towards the surface and, as for the residue-surface separations, the pattern in the orientations typically is similar to that of an $\alpha$-helix This is less clear towards the C-terminus, which is the region with lower $\alpha$-helix propensity (Figure 2). On the SAMoh surface these values of $\cos \theta$ are typically smaller suggesting that the hydrophilic surface has a weaker ordering effect.

As well as changing the average separations between polypeptide residues and surfaces, changing the surface hydrophobicity also affects the time-variation of these. From the timeseries of residue centre-of-mass-surface separations (Figure 9(a)) it can be seen that the hydrophobic surface leads to long lived (across the length of the simulations) contacts between the hIAPP and surface. These long-lived contacts greatly restrict the conformations that the protein adopts, leading to the restricted conformational ensemble compared to bulk solution.

On the SAMoh surface more variation in the contacts are seen. Notably there are transient desorptions of hIAPP from the SAMoh surface, which are not seen for the SAMch3 surface (Figure 9(b)), showing it binds more weakly onto the hydrophilic surface. This desorption also gives hIAPP more conformational freedom, allowing it to access a wider range of conformations. While Figure 9 shows data corresponding the $\beta=1$ replica, similar behaviour is seen in the demultiplexed trajectories (Figure S3), which correspond to single continuous trajectories. Note that the cluster analysis presented above is performed only for conformations where hIAPP is bound to the surface so a larger number of conformations found on the SAMoh surface compared to the SAMch3 surface is not due to desorbed conformations.

\section{CONCLUSIONS}

Using replica exchange molecular dynamics simulations the conformations of the fibril forming polypeptide, hIAPP, on different surfaces has been investigated. It has long been known that surfaces can affect the aggregation and assembly of proteins, in a surface dependent-manner. Previous experimental work has shown that fibril formation by hIAPP 
(a)
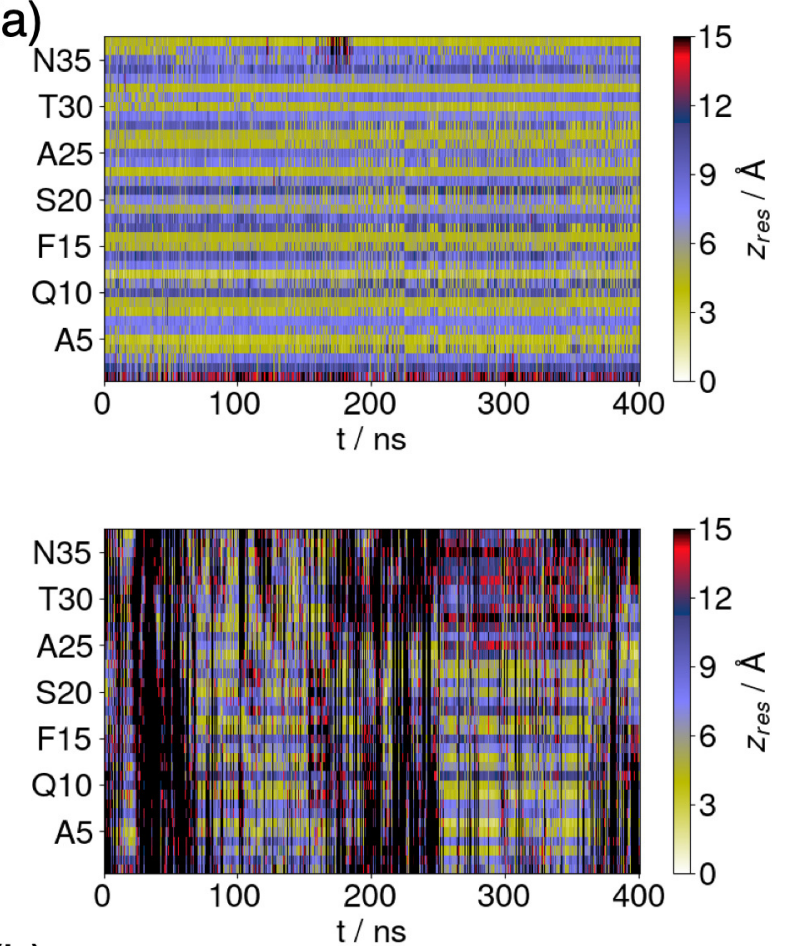

(b)

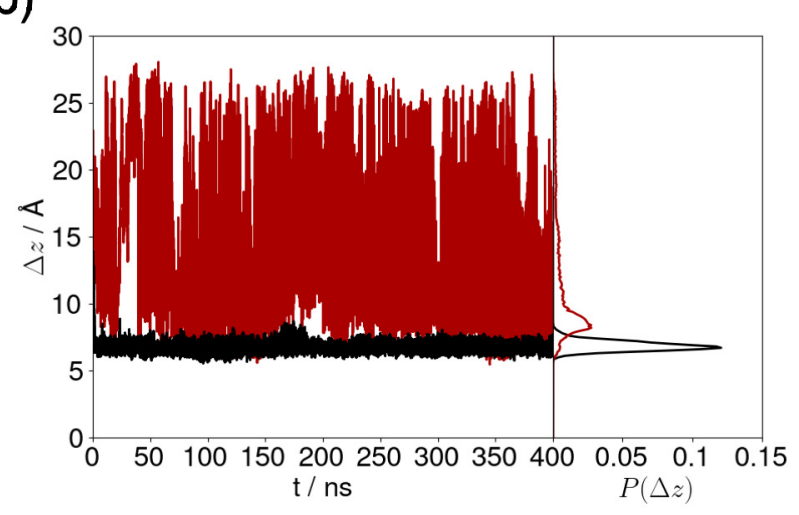

FIG. 9. (a) Residue centre-of-mass-surface separations for hIAPP on SAMch3 (top) and SAMoh (bottom) surfaces. (b) Polypeptide centre-of-mass-surface separations for hIAPP on SAMch3 (black) and SAMoh (red). Right hand panel shows polypeptide-surface separation probability histograms.

is enhanced on hydrophilic surfaces but inhibited on hydrophobic surfaces ${ }^{11}$. This is despite the fact that hIAPP concentration is higher on hydrophobic surfaces, indicating a decoupling between increased concentration and fibrillation rate. Knowledge of the differences in hIAPP conformation on these different surfaces is then important in understanding the relationship between concentration and aggregation. 
Both the secondary and tertiary structure of hIAPP are significantly different on a hydrophobic (SAMch3) surface to a hydrophilic (SAMoh) surface or in bulk solution. The hydrophobic surface leads to the formation of a largely $\alpha$-helical structure, seen both from explicit secondary structure analysis and from other measures of protein structure (number of $\alpha$-helical hydrogen bonds, dihedral offset). On the hydrophilic surface and in bulk solution the structure of hIAPP is similar and has a lower $\alpha$-helical content, with a variable region towards the C-terminus. This also leads to a more compact structure than on hydrophobic surface. Formation of $\alpha$-helix on the SAMch3 surface is driven by the affinity of the hydrophobic residues for the surface.

Changes to the surface chemistry also leads to hIAPP exhibiting different conformational ensembles. On the hydrophobic surface this is greatly restricted compared to the hydrophilic surface and bulk solution, with only 17 unique conformations found on the hydrophobic surface compared to 260 and 315 on the hydrophilic (SAMoh) surface and bulk solution respectively. Long-lived contacts between hydrophobic residues and the SAMch3 surface restricts the conformations that hIAPP can adopt, leading to a significantly reduced number of conformations. The protein is also strongly adsorbed onto the hydrophobic SAMch3 surface, whereas on the SAMoh surface transient desorption is seen. The desorption of the protein removes constraints on the protein conformation imposed by the surface, allowing it to adopt a wider range of conformations on the hydrophilic surface. This weaker binding and transient desorption also likely increases the diffusion rate of the protein on the hydrophilic surface, enhancing the fibrillation rate $^{8,61,62}$.

Comparison between the surface and bulk simulations shows that the surface adsorption alters the conformational ensemble in a surface dependent manner. Similar conformations are found in both the bulk simulations and on the SAMoh surface, suggesting that the conformational ensembles are similar in these environments. Greater differences are seen between bulk solution and the SAMch3 surface, indicating the hydrophobic surface templates the formation of a different set of conformations. Fibril-like conformations are also rarely found on the hydrophobic surface, with structures similar to those found in a micellar environment ${ }^{35}$ being adopted. This suggests that the increase in the fibrillation rate on hydrophilic surfaces is driven by the increased protein concentration, compared to bulk solution.

The adsorption of IDPs onto surfaces can be understood using models derived for protein 
binding and recognition ${ }^{63,64}$ On the hydrophilic surface the protein exhibits conformations that are largely similar to those found in bulk solution, although with different probabilities. This is similar to the conformational selection model of protein binding ${ }^{65}$. On the hydrophobic surface the protein exhibits induced fit behaviour ${ }^{66}$, where it largely adopts conformations different to those found in bulk solution. Previous simulations of intrinsically disordered proteins on gold surfaces ${ }^{17,32}$ have found both these types of behaviour; in this case it is shown that the same protein can exhibit different binding modes depending on the surface.

Notably the structure in solution is similar to that seen in previous simulations ${ }^{57}$. In that case the helical structure was found to be metastable compared to a less ordered structure. The difference with this work may be due to the charmm36m force field favouring more ordered structures; in a recent comparison of force fields for IAPP the charmm36 force field was found to give a more ordered structure than the charmm $22^{*}$ force field ${ }^{67}$.

More generally these results depend on the transferability of the charmm $36 \mathrm{~m}$ force field to surface simulations. While this is generally assumed and varients of the charmm force field have been used for a number of protein adsorption studies, this can only be tested through validation with experimental work and comparison with other force fields. The simulations also investigate only a limited timescale (400-600 ns). While consideration of the number of clusters suggest that the system has equilibrated in this period (Figure S2), longer simulations may uncover conformations not found in this work. Nonetheless the results of this work are consistent with experimental work ${ }^{11}$ and the formation of $\alpha$-helical structures

on hydrophobic surfaces and interfaces ${ }^{22,31,68}$ has been seen in a number of previous studies using a number of force fields. This suggests that, within the caveats above, molecular simulation can give insight into the role of surface chemistry on protein conformation and aggregation.

\section{ACKNOWLEDGEMENTS}

Computational facilities for this work were provided by the SFI/HEA funded Irish Centre for High End Computing. Atomic structures for hIAPP in fibrils were provided by Prof. Robert Tycko (NIH). The author wishes to thank Dr. Kieran Somers for useful discussions during this work. 


\section{SUPPORTING INFORMATION}

Details on simulation convergence and sampling. Analysis of demultiplexed trajectories. Sample simulation input files.

* david.cheung@nuigalway.ie

1 F. Chiti and C. M. Dobson, Annual Review of Biochemistry 75, 333 (2006), ISSN 0066-4154, URL http://www . annualreviews.org/doi/10.1146/annurev.biochem.75.101304.123901.

2 E. E. Erskine, C. E. MacPhee, and N. R. Stanley-Wall, Journal of Molecular Biology 430, 3642 (2018), ISSN 00222836, URL https://linkinghub.elsevier.com/retrieve/pii/ S0022283618308908.

3 C. Zhong, T. Gurry, A. A. Cheng, J. Downey, Z. Deng, C. M. Stultz, and T. K. Lu, Nature Nanotech 9, 858 (2014), ISSN 1748-3387, 1748-3395, URL http://www . nature.com/articles/ nnano.2014.199.

4 R. P. McGlinchey and J. C. Lee, Journal of Molecular Biology 430, 3696 (2018), ISSN 00222836, URL https://linkinghub.elsevier.com/retrieve/pii/S0022283618305904.

5 T. P. J. Knowles and R. Mezzenga, Advanced Materials 28, 6546 (2016), ISSN 15214095.

6 S. Bhattacharya, L. Xu, and D. Thompson, Wiley Interdisciplinary Reviews: Computational Molecular Science (2018), ISSN 17590884.

7 K. A. Burke, E. A. Yates, and J. Legleiter, Front. Neurol. 4, 17 (2013), ISSN 1664-2295, URL http://journal.frontiersin.org/article/10.3389/fneur.2013.00017/abstract.

8 A. Keller and G. Grundmeier, Applied Surface Science 506, 144991 (2020), ISSN 01694332, URL https://linkinghub.elsevier.com/retrieve/pii/S0169433219338085.

9 A. Keller, M. Fritzsche, Y. P. Yu, Q. Liu, Y. M. Li, M. Dong, and F. Besenbacher, ACS Nano 5, 2770 (2011), ISSN 19360851.

10 R. Hajiraissi, I. Giner, G. Grundmeier, and A. Keller, Langmuir 33, 372 (2016), ISSN 0743-7463, URL http://pubs.acs.org/doi/abs/10.1021/acs. langmuir.6b03288.

11 R. Hajiraissi, M. Hanke, Y. Yang, B. Duderija, A. Gonzalez Orive, G. Grundmeier, and A. Keller, Langmuir 34, 3517 (2018), ISSN 15205827. 
12 T. Ando, Current Opinion in Structural Biology 28, 63 (2014), ISSN 0959440X, URL https: //linkinghub.elsevier.com/retrieve/pii/S0959440X14000906.

13 L. Feng, H. Watanabe, P. Molino, G. G. Wallace, S. L. Phung, T. Uchihashi, and M. J. Higgins, Journal of Molecular Biology 431, 2687 (2019), ISSN 00222836, URL https://linkinghub. elsevier.com/retrieve/pii/S0022283619302529.

14 S. Hosseinpour, S. J. Roeters, M. Bonn, W. Peukert, S. Woutersen, and T. Weidner, Chem. Rev. p. acs.chemrev.9b00410 (2020), ISSN 0009-2665, 1520-6890, URL https://pubs.acs. org/doi/abs/10.1021/acs . chemrev.9b00410.

15 L. Fu, Z. Wang, B. T. Psciuk, D. Xiao, V. S. Batista, and E. C. Y. Yan, J. Phys. Chem. Lett. 6, 1310 (2015), ISSN 1948-7185, URL https://pubs.acs.org/doi/10.1021/acs.jpclett. $5 \mathrm{~b} 00326$.

16 J. Tan, J. Zhang, Y. Luo, and S. Ye, J. Am. Chem. Soc. 141, 1941 (2019), ISSN 0002-7863, 1520-5126, URL https://pubs.acs.org/doi/10.1021/jacs.8b08537.

17 L. Bellucci, A. Ardèvol, M. Parrinello, H. Lutz, H. Lu, T. Weidner, and S. Corni, Nanoscale 8, 8737 (2016), ISSN 2040-3364, 2040-3372, URL http://xlink.rsc.org/?DOI=C6NR01539E.

18 M. Ozboyaci, D. B. Kokh, S. Corni, and R. C. Wade, Quarterly Reviews of Biophysics 49, 1 (2016), ISSN 14698994.

19 K. Ley, A. Christofferson, M. Penna, D. Winkler, S. Maclaughlin, and I. Yarovsky, Frontiers in Molecular Biosciences 2, 1 (2015), ISSN 2296-889X, URL http://journal.frontiersin.org/ Article/10.3389/fmolb.2015.00064/abstract.

20 K. Kubiak-Ossowska, P. A. Mulheran, and W. Nowak, Journal of Physical Chemistry B 118, $9900(2014)$.

21 D. Mallinson, D. L. Cheung, D. Simionesie, A. B. Mullen, Z. J. Zhang, and D. A. Lamprou, Journal of Biomedical Materials Research - Part A 105, 737 (2017), ISSN 15524965.

22 M. Deighan and J. Pfaendtner, Langmuir 29, 7999 (2013).

23 Q. Wang, C. Zhao, J. Zhao, J. Wang, J.-C. Yang, X. Yu, and J. Zheng, Langmuir 26, 3308 (2010), ISSN 0743-7463, 1520-5827, URL https://pubs.acs.org/doi/10.1021/la903070y.

24 S.-T. Wang, Y. Lin, N. Todorova, Y. Xu, M. Mazo, S. Rana, V. Leonardo, N. Amdursky, C. D. Spicer, B. D. Alexander, et al., Chemistry of Materials 29, 1550 (2017), ISSN 0897-4756, 1520-5002, URL http://pubs.acs.org/doi/10.1021/acs.chemmater.6b04144. 
25 S.-g. Kang, H. Li, T. Huynh, F. Zhang, Z. Xia, Y. Zhang, and R. Zhou, ACS Nano 6, 9276 (2012), ISSN 1936-0851, 1936-086X, URL https://pubs .acs.org/doi/10.1021/nn303740j.

26 S.-g. Kang, T. Huynh, Z. Xia, Y. Zhang, H. Fang, G. Wei, and R. Zhou, J. Am. Chem. Soc. 135, 3150 (2013), ISSN 0002-7863, 1520-5126, URL https://pubs.acs.org/doi/10.1021/ ja310989u.

27 B. Dai, S.-g. Kang, T. Huynh, H. Lei, M. Castelli, J. Hu, Y. Zhang, and R. Zhou, Proceedings of the National Academy of Sciences 110, 8543 (2013), ISSN 0027-8424, 1091-6490, URL http: //www.pnas.org/cgi/doi/10.1073/pnas.1220711110.

28 A. Laio and F. L. Gervasio, Reports on Progress in Physics 71, 126601 (2008), ISSN 0034-4885, URL http://stacks.iop.org/0034-4885/71/i=12/a=126601.

29 D. J. Earl and M. W. Deem, Physical Chemistry Chemical Physics 7, 3910 (2005).

30 D. Granata, F. Baftizadeh, J. Habchi, C. Galvagnion, A. De Simone, C. Camilloni, A. Laio, and M. Vendruscolo, Scientific Reports 5, 1 (2015), ISSN 20452322, URL http://dx.doi.org/ 10.1038/srep15449.

31 D. L. Cheung, J. Chem. Phys. 151, 064706 (2019), ISSN 0021-9606, 1089-7690, URL http: //aip.scitation.org/doi/10.1063/1.5100253.

32 L. Bellucci, G. Bussi, R. Di Felice, and S. Corni, Nanoscale 9, 2279 (2017), ISSN 2040-3364, URL http://xlink.rsc.org/?DOI=C6NR06010B.

33 J. Schneider and L. Colombi Ciacchi, Journal of the American Chemical Society 134, 2407 (2012), ISSN 00027863.

34 L. B. Wright, J. P. Palafox-Hernandez, P. M. Rodger, S. Corni, and T. R. Walsh, Chem. Sci. 6, 5204 (2015), ISSN 2041-6520, URL http://xlink.rsc.org/?DOI=C5SC00399G.

35 S. M. Patil, S. Xu, S. R. Sheftic, and A. T. Alexandrescu, Journal of Biological Chemistry 284, 11982 (2009), ISSN 00219258.

36 A. D. MacKerell, D. Bashford, R. L. Dunbrack, J. D. Evanseck, M. J. Field, S. Fischer, J. Gao, H. Guo, S. Ha, D. Joseph-McCarthy, et al., The Journal of Physical Chemistry B 102, 3586 (1998), ISSN 1520-6106, URL http://dx.doi.org/10.1021/jp973084f.

37 J. Huang, S. Rauscher, G. Nawrocki, T. Ran, M. Feig, B. L. De Groot, H. Grubmüller, and A. D. Mackerell, Nature Methods 14, 71 (2016), ISSN 1548-7091, URL http://dx.doi.org/ $10.1038 /$ nmeth .4067 .

38 W. L. Jorgensen, J. Chandrasekhar, and J. D. Madura, J. Chem. Phys. 79, 926 (1983). 
39 P. Mark and L. Nilsson, Journal of Physical Chemistry A 105, 9954 (2001).

40 K. Vanommeslaeghe, E. Hatcher, C. Acharya, S. Kundu, S. Zhong, J. Shim, E. Darian, O. Guvench, P. Lopes, I. Vorobyov, et al., J. Comput. Chem. 31, 671 (2009), ISSN 01928651, 1096987X, URL http://doi.wiley.com/10.1002/jcc.21367.

41 P. Liu, B. Kim, R. A. Friesner, and B. J. Berne, Proceedings of the National Academy of Sciences of the United States of America 102, 13749 (2005), ISSN 0027-8424, URL http: //www.pnas.org/cgi/content/long/102/39/13749.

42 L. Wang, R. A. Friesner, and B. J. Berne, Journal of Physical Chemistry B 115, 9431 (2011), ISSN 15205207.

43 Y. Sugita and Y. Okamoto, Chemical Physics Letters 314, 141 (1999), ISSN 1064-3745, URL http://www.ncbi.nlm.nih.gov/pubmed/16957325.

44 G. Bussi, D. Donadio, and M. Parrinello, Journal of Chemical Physics 126, 014101/1 (2007), ISSN 00219606.

45 M. Parrinello and A. Rahman, Journal of Applied Physics 52, 7182 (1981), ISSN 00218979.

46 U. Essmann, L. Perera, M. L. Berkowitz, T. Darden, H. Lee, and L. G. Pedersen, Journal of Computational Physics 103, 8577 (1995).

47 B. Hess, H. Bekker, H. J. C. Berendsen, and J. G. E. M. Fraaije, Journal of computational chemistry 18, 1463 (1997).

48 D. Van Der Spoel, E. Lindahl, B. Hess, G. Groenhof, A. E. Mark, and H. J. C. Berendsen, Journal of Computational Chemistry 26, 1701 (2005), ISSN 01928651.

49 B. Hess, C. Kutzner, D. van der Spoel, and E. Lindahl, J. Chem. Theory Comput. 4, 435 (2008), ISSN 15499618, URL http://pubs.acs.org/doi/abs/10.1021/ct700301q.

50 M. J. Abraham, T. Murtola, R. Schulz, S. Páll, J. C. Smith, B. Hess, and E. Lindahl, SoftwareX 1-2, 19 (2015), ISSN 23527110, URL https://linkinghub.elsevier.com/retrieve/ $\mathrm{pii/S2352711015000059.}$

51 G. A. Tribello, M. Bonomi, D. Branduardi, C. Camilloni, and G. Bussi, Computer Physics Communications 185, 604 (2014), ISSN 00104655, URL http://dx.doi.org/10.1016/j.cpc. 2013.09 .018$.

52 N. Michaud-Agrawal, E. J. Denning, T. B. Woolf, and O. Beckstein, Journal of computational chemistry 32, 2319 (2011), ISSN 1096-987X. 
53 W. Humphrey, A. Dalke, and K. Schulten, Journal of Molecular Graphics 14, 33 (1996), ISSN 02637855, URL http://dx.doi.org/10.1016/0263-7855(96)00018-5.

54 M. Heinig and D. Frishman, Nucleic Acids Research 32, 500 (2004), ISSN 03051048.

55 X. Daura, K. Gademann, B. Jaun, D. Seebach, W. F. Van Gunsteren, and A. E. Mark, Angew. Chemie Int. Ed. 38, 236 (1999), ISSN 1433-7851, URL http://doi.wiley.com/10.1002/(SICI) 1521-3773(19990115)38:1/2\%7B\%25\%7D3C236: : AID-ANIE236\%7B\%25\%7D3E3.0.CD;2-M.

56 A. De Simone, C. Kitchen, A. H. Kwan, M. Sunde, C. M. Dobson, D. Frenkel, A. D. Simone, C. Kitchen, A. H. Kwan, M. Sunde, et al., Proceedings of the National Academy of Sciences of the United States of America 109, 6951 (2012), ISSN 0027-8424.

57 A. S. Reddy, L. Wang, S. Singh, Y. L. Ling, L. Buchanan, M. T. Zanni, J. L. Skinner, and J. J. de Pablo, Biophysical Journal 99, 2208 (2010), ISSN 00063495, URL https://linkinghub. elsevier.com/retrieve/pii/S0006349510008593.

58 J.-J. Ho, A. Ghosh, T. O. Zhang, and M. T. Zanni, J. Phys. Chem. A 122, 1270 (2018), ISSN 1089-5639, 1520-5215, URL http://pubs.acs.org/doi/10.1021/acs.jpca.7b11934.

59 S. Luca, W.-M. Yau, R. Leapman, and R. Tycko, Biochemistry 46, 13505 (2007), ISSN 00062960, 1520-4995, URL https://pubs.acs.org/doi/10.1021/bi701427q.

60 R. Hajiraissi, M. Hanke, A. Gonzalez Orive, B. Duderija, U. Hofmann, Y. Zhang, G. Grundmeier, and A. Keller, ACS Omega 4, 2649 (2019), ISSN 2470-1343, 2470-1343, URL https: //pubs.acs.org/doi/10.1021/acsomega.8b03028.

61 L. Shen, T. Adachi, D. Vanden Bout, and X.-Y. Zhu, J. Am. Chem. Soc. 134, 14172 (2012), ISSN 0002-7863, 1520-5126, URL https://pubs.acs.org/doi/10.1021/ja305398f.

62 Y.-C. Lin, C. Li, and Z. Fakhraai, Langmuir 34, 4665 (2018), ISSN 0743-7463, 1520-5827, URL https://pubs.acs.org/doi/10.1021/acs. langmuir.7b02744.

63 P. E. Wright and H. J. Dyson, Current Opinion in Structural Biology 19, 31 (2009), ISSN 0959440X, URL https://linkinghub.elsevier.com/retrieve/pii/S0959440X08001796.

64 N. London, D. Movshovitz-Attias, and O. Schueler-Furman, Structure 18, 188 (2010), ISSN 09692126, URL https://linkinghub.elsevier.com/retrieve/pii/S096921260900478X.

65 S. Kumar, B. Ma, C.-J. Tsai, N. Sinha, and R. Nussinov, Protein Science 9, 10 (2008), ISSN 09618368, URL http://doi.wiley.com/10.1110/ps.9.1.10.

66 D. E. Koshland, Proc. Natl. Acad. Sci. 44, 98 (1958). 
67 E. Peng, N. Todorova, and I. Yarovsky, PLOS ONE 12, e0186219 (2017), ISSN 1932-6203, URL http://dx.plos.org/10.1371/journal.pone.0186219.

68 C. Dalgicdir, C. Globisch, C. Peter, and M. Sayar, PLOS Computational Biology 11, e1004328 (2015), ISSN 1553-7358, URL http://dx.plos.org/10.1371/journal.pcbi.1004328. 


\title{
Supporting Information for 'Effect of surface chemistry on IAPP conformation'
}

\author{
David L. Cheung \\ School of Chemistry, National University of Ireland Galway, Galway, Ireland*
}




\section{S1. CONVERGENCE OF REST SIMULATIONS}

Motion of trajectories between replicas can be monitored through the variation of the REST scaling parameter $\left(\beta_{i}\right)$ for different replicas (Figure S1). As can be seen the replicas explore different values of $\beta_{i}$. Shown in Table S1 are the acceptance rates for the simulations. In all cases the acceptance rates are above $20 \%$ for all simulations and all pairs of replicas.

SAMch3

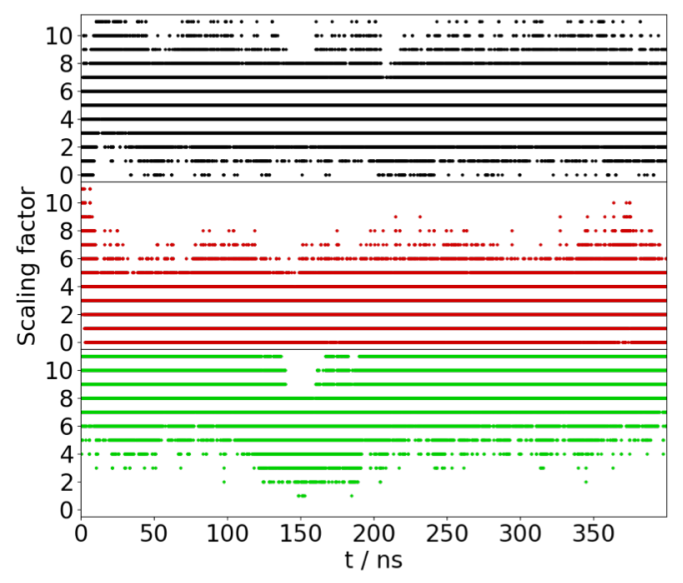

SAMoh

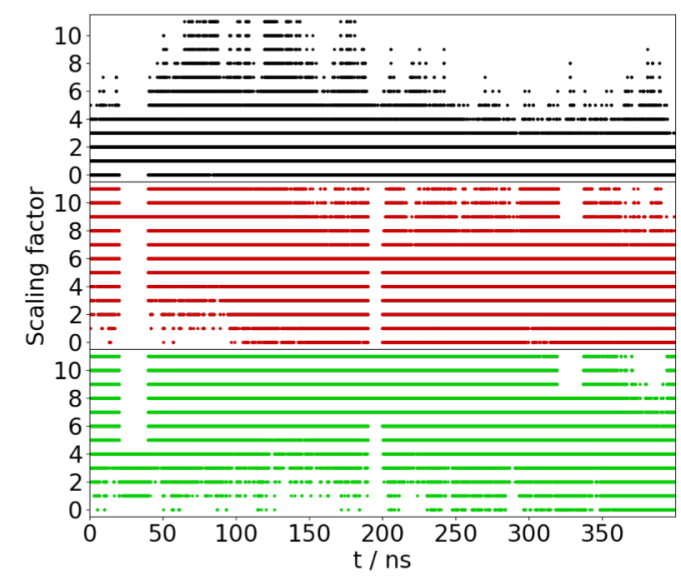

Solution

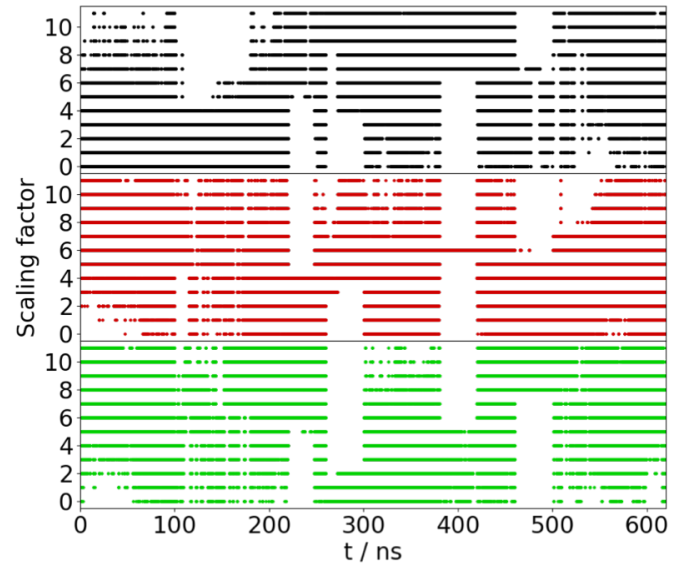

FIG. S1: Plot of scaling factor $\left(i\right.$ denotes $\beta_{i}$ ) for hIAPP. Graphs show (from top to bottom) replicas with $i=0,6$, and 11 at $t=0$. 
Simulation $\quad 0 \leftrightarrow 1 \quad 1 \leftrightarrow 2 \quad 2 \leftrightarrow 3 \quad 3 \leftrightarrow 4 \quad 4 \leftrightarrow 5 \quad 5 \leftrightarrow 6 \quad 6 \leftrightarrow 7 \quad 7 \leftrightarrow 8 \quad 8 \leftrightarrow 9 \quad 9 \leftrightarrow 10 \quad 10 \leftrightarrow 11$

SAMch3 $36.7 \% 40.8 \% 29.2 \% 31.2 \% 29.8 \% 36.4 \% 43.6 \% 35.9 \% 40.2 \% 45.2 \% 34.8 \%$

SAMoh $\quad 34.0 \% 42.0 \% 39.1 \% 44.2 \% 37.8 \% 42.0 \% 45.2 \% 40.0 \% 43.9 \% 45.1 \% 39.5 \%$

Solution $\quad 26.0 \% 34.4 \% 27.7 \% 38.2 \% 29.6 \% 36.4 \% 27.1 \% 28.6 \% 34.6 \% 40.3 \% 33.2 \%$

TABLE S1: Acceptance rates for REST simulations.

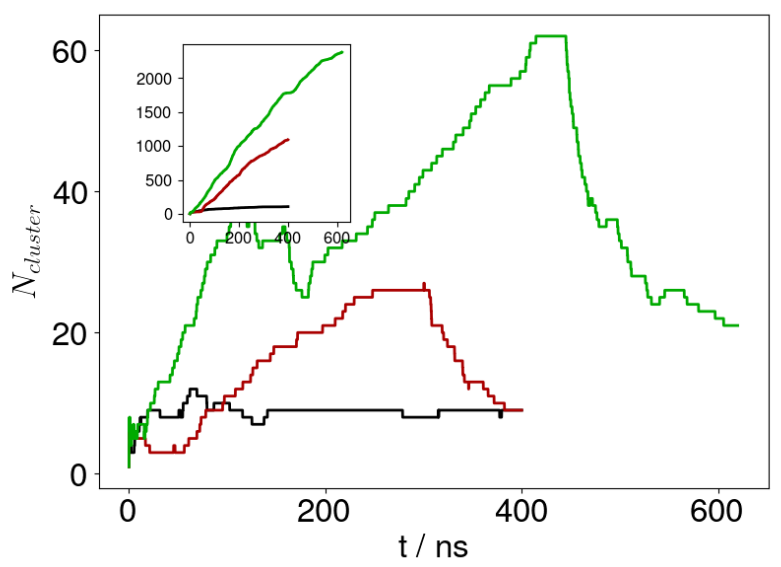

FIG. S2: Variation in number of clusters found with simulation time. Main figure shows clusters with free energy within $3 \mathrm{kcal}$ mol-1 of the ground state, inset shows total number of clusters. Black, red, and green denote SAMch3, SAMoh and solution simulations respectively. 


\section{S2. DEMULTIPLEXED TRAJECTORY ANALYSIS}

Shown in Figure S3 are the protein-surface separations for selected replicas (continuous trajectories). For the SAMch3 surface the protein remains closely bound to the surface for all the replicas. On the SAMoh surface transient desorptions are seen for all replicas.
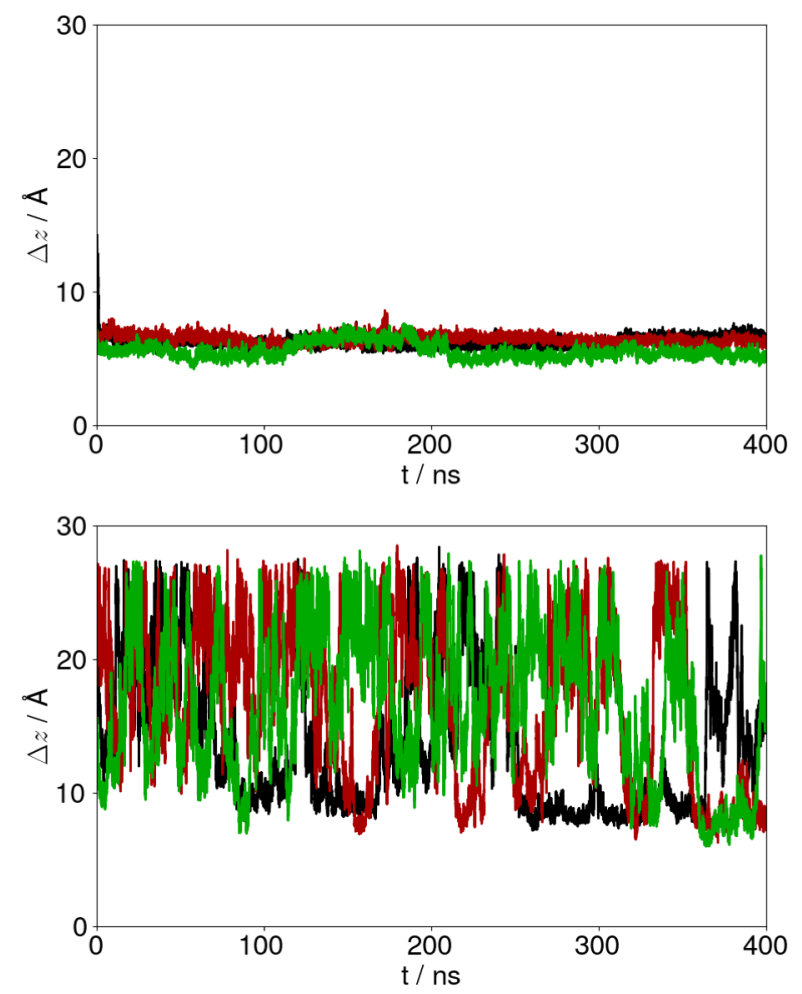

FIG. S3: Protein-surface separations from demultiplexed trajectories for SAMch3 (top) and SAMoh (bottom) surfaces. Black, red, and green lines denote replicas with $i=0,6$, and 11 at $t=0$.

Shown in Figures S4-S4 is the time variation of the secondary structure across the simulations. In many cases changes to the secondary structure are clearly seen, again showing that sampling of different protein conformations is occurring within each trajectory. 

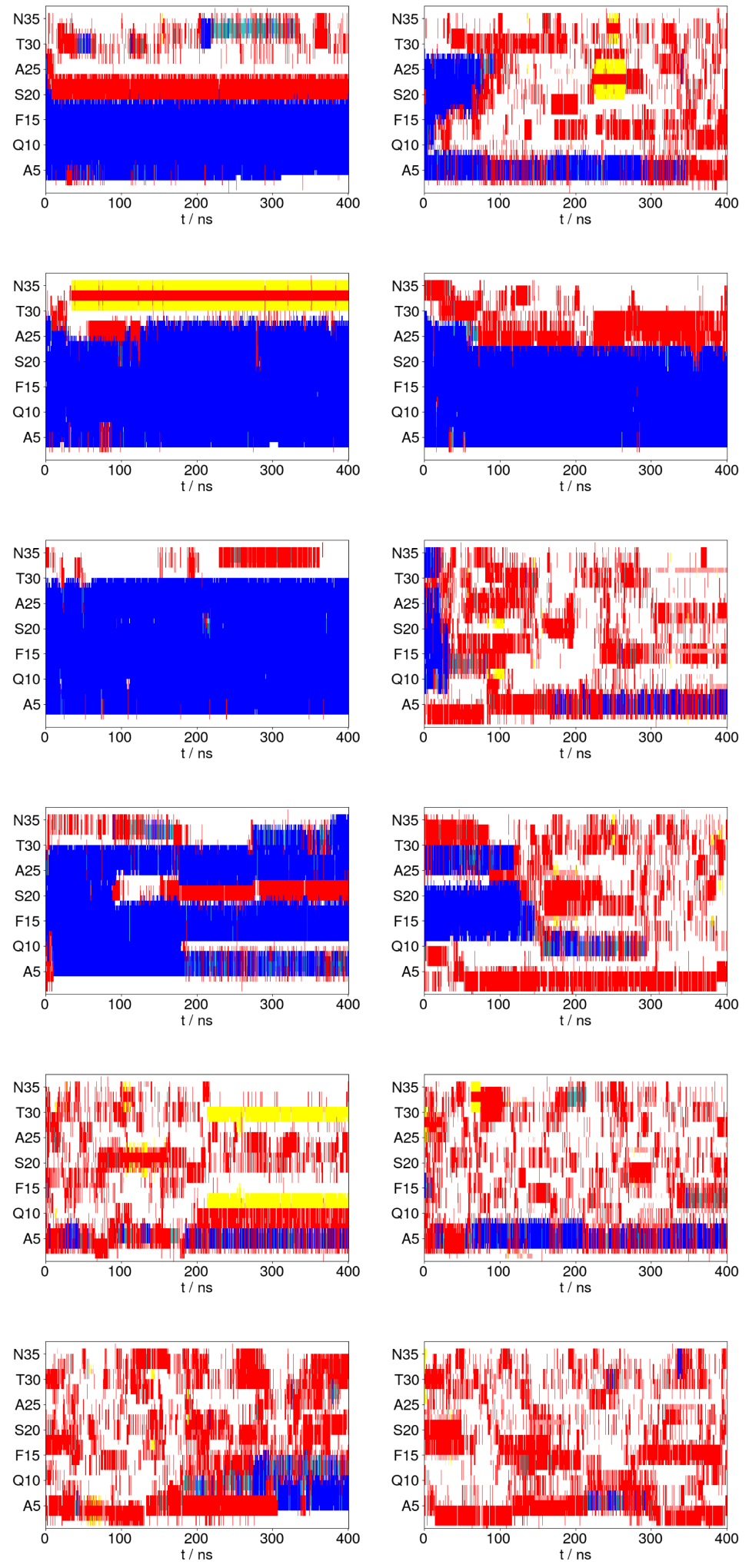

FIG. S4: Secondary structure evolution for demultiplexed trajectories for hIAPP on SAMch3 surface. 

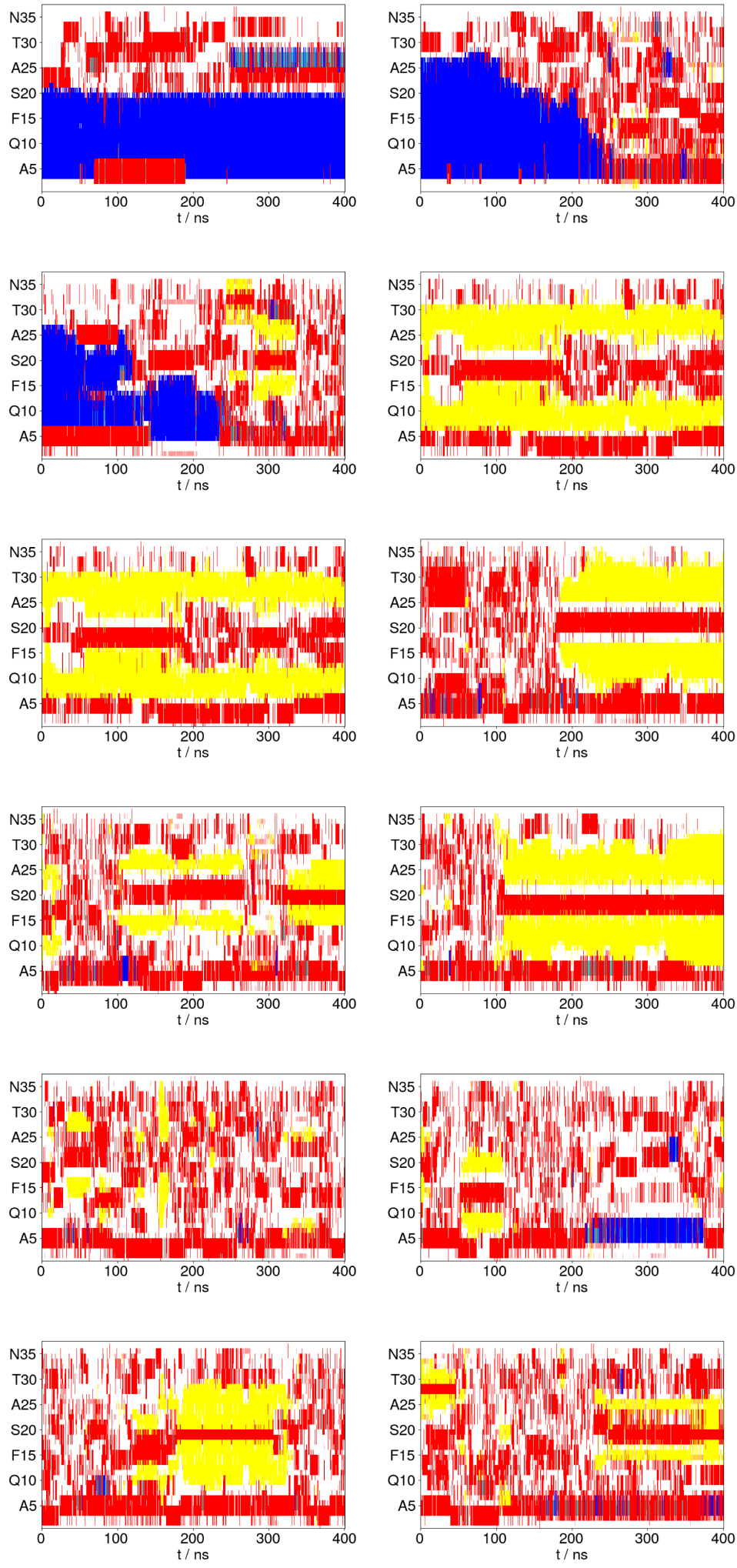

FIG. S5: Secondary structure evolution for demultiplexed trajectories for hIAPP on SAMoh surface. 

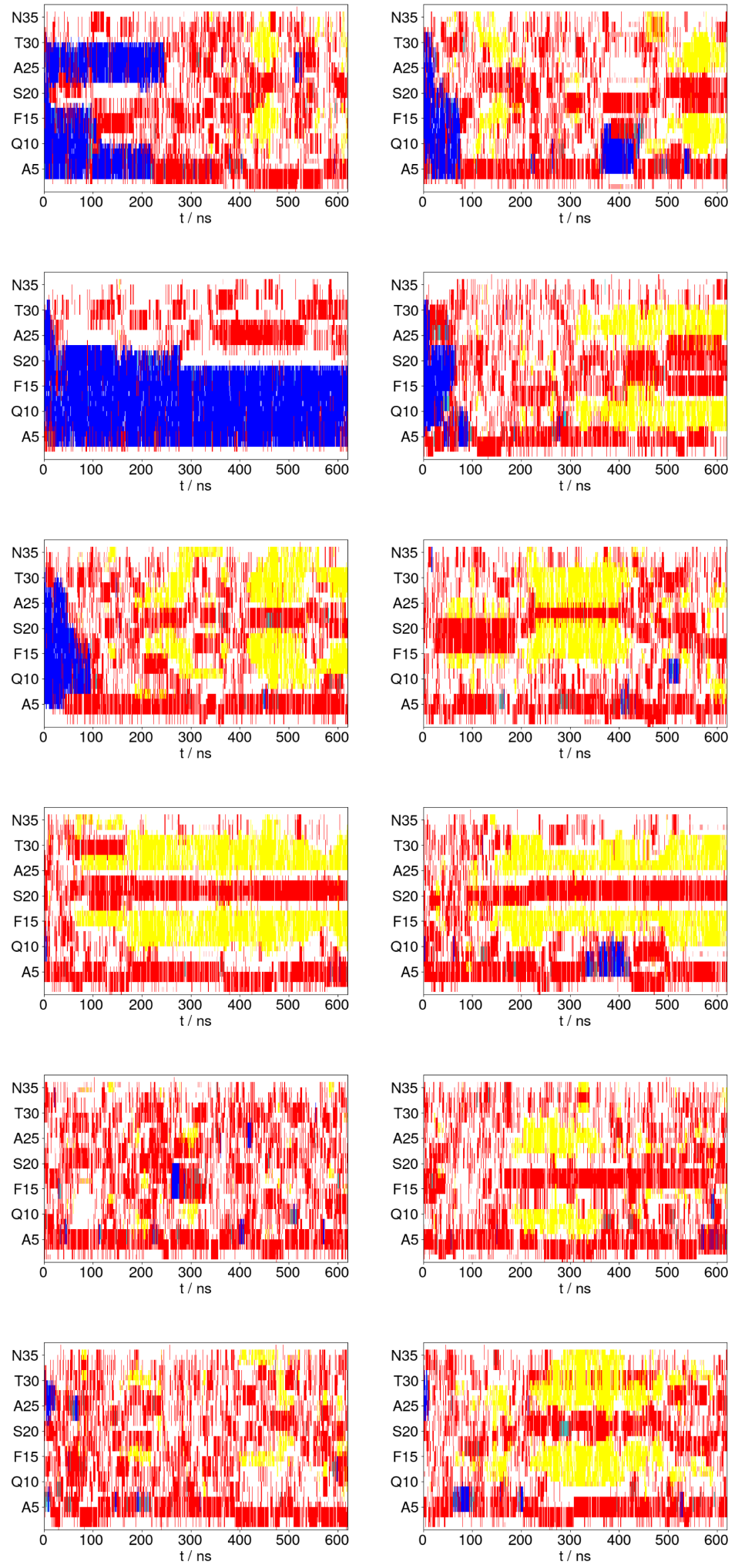

FIG. S6: Secondary structure evolution for demultiplexed trajectories for hIAPP in bulk solution. 


\section{S3. SOLUTION CLUSTER POPULATIONS IN SURFACE SIMULATIONS}

Table S2 are the populations of solution-like clusters found in the surface simulations.

\begin{tabular}{cccc}
\hline Surface & Cluster & Prob. & FE \\
\hline & 73 & 0.050 & 3.85 \\
SAMch3 & 263 & 0.095 & 5.16 \\
& NC & 0.855 & - \\
\hline & 77 & 0.040 & 3.85 \\
& 215 & 0.613 & 5.16 \\
& 223 & 0.039 & 5.16 \\
& 287 & 0.014 & 5.16 \\
SAMoh & 297 & 0.016 & 5.16 \\
& 305 & 0.033 & 5.16 \\
& 309 & 0.037 & 5.16 \\
& NC & 0.127 & - \\
\hline
\end{tabular}

TABLE S2: Populations of clusters from bulk solution found in surface simulations. $P$ is the probability of that conformation being found in the surface simulations. $F E$ is the free energy of that conformation in the bulk simulations. NC are conformations not found in bulk simulations. 